\title{
Accuracy of soft tissue balancing in total knee arthroplasty using surgeon-defined assessment versus a gap-balancer or electronic sensor
}

\author{
Ran Zhao, Yanqing Liu* and Hua Tian*
}

\begin{abstract}
Background: Soft tissue balancing is essential for the success of total knee arthroplasty (TKA) and is mainly dependent on surgeon-defined assessment (SDA) or a gap-balancer (GB). However, an electronic sensor has been developed to objectively measure the gap pressure. This study aimed to evaluate the accuracy of soft tissue balancing using SDA and GB compared with a sensor.

Methods: Forty-eight patients undergoing TKA (60 knees) were prospectively enrolled. Soft tissue balancing was sequentially performed using SDA, a GB, and an electronic sensor. We compared the SDA, GB, and sensor data to calculate the sensitivity, specificity, and accuracy at $0^{\circ}, 45^{\circ}, 90^{\circ}$, and $120^{\circ}$ flexion. Cumulative summation (CUSUM) analysis was performed to assess the surgeon's performance during the sensor introductory phase.

Results: The sensitivity of SDA was $63.3 \%, 68.3 \%, 80.0 \%$, and $80.0 \%$ at $0^{\circ}, 45^{\circ}, 90^{\circ}$, and $120^{\circ}$, respectively. The accuracy of the $\mathrm{GB}$ compared with sensor data was $76.7 \%$ and $71.7 \%$ at $0^{\circ}$ and $90^{\circ}$, respectively.

Cohen's kappa coefficient for the accuracy of the GB was 0.406 at $0^{\circ}$ (moderate agreement) and 0.227 at $90^{\circ}$ (fair agreement). The CUSUM $0^{\circ}$ line achieved good prior performance at case 45 , CUSUM $90^{\circ}$ and $120^{\circ}$ showed a trend toward good prior performance, while CUSUM $45^{\circ}$ reached poor prior performance at case 8.

Conclusion: SDA was a poor predictor of knee balance. GB improved the accuracy of soft tissue balancing, but was still less accurate than the sensor, particularly for unbalanced knees. SDA improved with ongoing use of the sensor, except at $45^{\circ}$ flexion.
\end{abstract}

Keywords: Total knee arthroplasty, Sensor, Gap-balancer, Learning curve

\section{Introduction}

Total knee arthroplasty (TKA) is an effective treatment for severe knee diseases, and soft tissue balance is a key factor in achieving a successful outcome [1,2]. Improper soft tissue balance may result in pain, stiffness, instability, or even polyethylene wear, leading to revision knee

\footnotetext{
* Correspondence: liuyanqing71@126.com; huatian@bjmu.edu.cn Peking University Third Hospital, Department of Orthopaedics, Engineering Research Center of Bone and Joint Precision Medicine, Ministry of Education, 49 North Garden Road, Beijing 100191, Haidian District, China
}

surgery [2-4]. Balancing is often determined using surgeon-defined assessment (SDA), which depends on operative experience and lacks an objective and quantitative standard. A gap-balancer (GB) is used to calculate the difference between the internal and external space. However, the variation between surgeons in the strength that they use to extend the gap may result in measurement errors. Furthermore, the distraction gap measurement is only accurate to $1 \mathrm{~mm}$, and can only be measured at $0^{\circ}$ and $90^{\circ}$ flexion [5].

(c) The Author(s). 2021 Open Access This article is licensed under a Creative Commons Attribution 4.0 International License, which permits use, sharing, adaptation, distribution and reproduction in any medium or format, as long as you give appropriate credit to the original author(s) and the source, provide a link to the Creative Commons licence, and indicate if changes were made. The images or other third party material in this article are included in the article's Creative Commons licence, unless indicated otherwise in a credit line to the material. If material is not included in the article's Creative Commons licence and your intended use is not permitted by statutory regulation or exceeds the permitted use, you will need to obtain permission directly from the copyright holder. To view a copy of this licence, visit http://creativecommons.org/licenses/by/4.0/ The Creative Commons Public Domain Dedication waiver (http://creativecommons.org/publicdomain/zero/1.0/) applies to the data made available in this article, unless otherwise stated in a credit line to the data. 
Sensor can objectively measure the intercompartmental loads of the knee during TKA, previous study compared sensor as a reference to SDA during soft tissue balancing [6]. SDA is reportedly a poor predictor of soft tissue balance compared with the sensor and is even worse at larger knee flexion angles [6]. The purpose of this study was to (1) evaluate the compartment loads throughout the knee range of motion in the sensorbalanced group and determine the sensitivity of SDA; (2) evaluate the sensitivity, specificity, and accuracy of the GB compared with the sensor; (3) verify that SDA of knee balance improves with the use of a sensor.

\section{Material and methods}

\section{Study cohort}

With the approval of the institutional ethics committee, data were prospectively collected from patients who underwent unilateral or bilateral TKA in our hospital from December 2018 to December 2019. Sensors were used in all cases. All patients provided written informed consent for study participation. The inclusion criteria were (1) osteoarthritis; (2) knee varus or valgus of less than $25^{\circ}$ and flexion contracture of less than $25^{\circ}$. The exclusion criteria were (1) rheumatoid arthritis, traumatic osteoarthritis, or prior osteotomy around the knee; (2) joint replacement with severe varum/varus/flexion contracture or bone deformity that required an allograft, constrained implants, or mental augmentation.

A total of 48 patients (60 knees) were included (31 left knees and 29 right knees). The average patient age was 66 years (range, $56-76$ years), and the average varus deformity angle was $12.6^{\circ}$ (range, $5-25^{\circ}$ ). The Genesis II cruciate-retaining implant (Smith \& Nephew, London, UK) was used for all operations. All surgeries were performed by the same senior knee arthroplasty surgeon, who performs approximately 300 to 350 knee surgeries per year and had not used the sensor technology prior to these 60 surgeries.

\section{Surgical technique}

A medial parapatellar approach was used for all surgeries. The deep part of the medial collateral ligament was retained and turned over the patella. The femoral resection was done using an intramedullary guide, while the tibial resection was done using an extramedullary guide to restore a neutral mechanical axis of the lower limb. Standard cutting blocks were used to complete the femoral and tibial preparations, and a spacer was used to guide the soft tissue balancing. A standard trial articular insert was used to determine the appropriate insert thickness. SDA was performed with the joint in four positions: $0^{\circ}, 45^{\circ}, 90^{\circ}$, and $120^{\circ}$ of flexion. The test was standardized by imparting varus and valgus stress on the knee, with one hand applying maximal stress to the tibia and the other hand stabilizing the femur. During SDA, the balance of collateral ligament tension was determined by visual and tactile assessments of the medial and lateral openings. Soft tissue release was carried out until the balance was considered satisfactory.

After the SDA was performed, the balance was checked using a GB and a wireless electronic pressure sensor insert with the same thickness and size (Yiemed Co. Ltd., Shandong, China) (Fig. 1). A GB with $20 \mathrm{lb}$ of force was used to open the knee joint space, and the difference between the medial and lateral compartments was measured at $0^{\circ}$ and $90^{\circ}$ of knee flexion. The sensor data were recorded with the limb held in neutral alignment to prevent the weight of the limb from stressing or distracting the joint. The sensor was used to determine the pressure in the medial and lateral components at knee flexion angles of $0^{\circ}, 45^{\circ}, 90^{\circ}$, and $120^{\circ}$. The data of each gap is measured three times by sensor, and the average value is taken to determine intraobserver agreement. Soft tissue release was then undertaken if required based on the sensor data. Once the knee was well balanced, a fully cemented cruciate-retaining knee prosthesis was implanted in all cases.

The Knee Society Score (KSS) and the Western Ontario and McMaster Universities (WOMAC) were recorded preoperatively and postoperatively. The KSS score consists of a functional and clinical subscale, with both a maximum score of 100 points. The WOMAC score consists of pain, stiffness and physical function, 0 point is the best and 96 points is the worst.

Definitions of knee balance using the three methods:

1. SDA: moderate tension of the medial and lateral ligaments, and a side-to-side difference of no more than $2 \mathrm{~mm}$ at each knee flexion angle when inserting the trial implants.

2. GB: a gap difference between the medial and lateral compartments at $0^{\circ}$ of knee flexion of less than 1 $\mathrm{mm}$, and a balance of 1-4 mm larger on the lateral side than the medial side at $90^{\circ}$ flexion. There was no femoral external rotation in the GII femoral prosthesis osteotomy, and the standard flexion gap was $2.5 \mathrm{~mm}$ wider on the lateral side than on the medial side.

3. Sensor: an intercompartmental pressure difference (ICPD) between the medial and lateral compartments of $30 \mathrm{~N}$ or less at each flexion angle. The pressure in the medial and lateral compartments was $0-100 \mathrm{~N}$.

\section{Statistical analysis}

The sensitivity of the SDA was calculated in comparison with the sensor data. The accuracy, sensitivity, specificity, and predictive values were calculated for the GB 

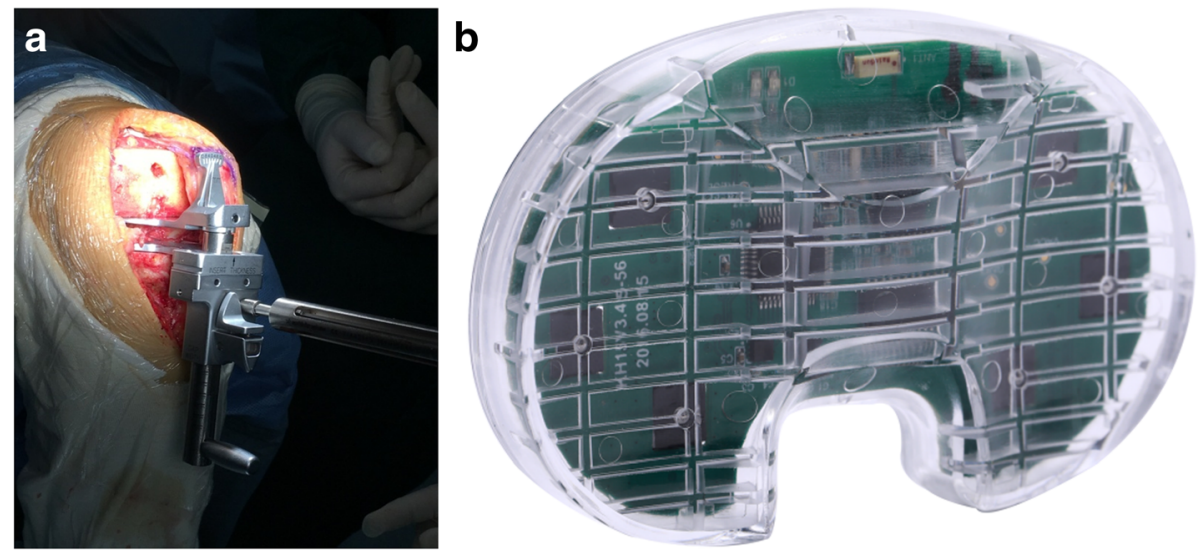

Fig. 1 a The gap-balancer for the Genesis II prosthesis. b The knee joint pressure sensor with a shape that conforms to the Genesis II prosthesis

compared with the sensor data. Cohen's kappa coefficient was calculated to determine the chance-corrected proportional agreement as slight (Cohen's kappa coefficient 0.01-0.20), fair (Cohen's kappa coefficient 0.210.40), moderate (Cohen's kappa coefficient 0.41-0.60), substantial (Cohen's kappa coefficient 0.61-0.80), and almost perfect (Cohen's kappa coefficient 0.81-0.99) [7]. Statistical significance was set at $P<0.05$.

Cumulative summation (CUSUM) is a sequential analysis method used to detect changes in performance and analyze the learning curve of a new technology [8]. CUSUM analysis was used to determine whether gap balance was achieved at $0^{\circ}, 45^{\circ}, 90^{\circ}$, and $120^{\circ}$ flexion. CUSUM charts were created to display the CUSUM values against the number of procedures. The CUSUM graph decreases with each success, and a horizontal or downward-sloping line indicates an acceptable performance. Conversely, the CUSUM graph increases with each failure, and an upward-sloping line indicates an unacceptable performance. We used horizontal control lines to help interpret the acceptability of the performance. If the CUSUM line crossed the acceptable control line from above, this indicated good prior performance (GPP). If the CUSUM line crossed the unacceptable control line from below, this indicated poor prior performance (PPP) [9].

Statistical analysis was performed using SPSS 20 (IBM SPSS Statistics for Windows, version 22.0; IBM Corp., Armonk, NY).

\section{Results}

Intraobserver variation of the sensor data assessed by calculating the intraclass correlation coefficient (ICC) was from 0.854 to 0.946 . No statistically significant differences in agreement or reliability of intraobserver measurements were found.

\section{Sensitivity and compartment loads of SDA}

In the 60 knees, the sensitivities of SDA compared with the sensor data at $0^{\circ}, 45^{\circ}, 90^{\circ}$, and $120^{\circ}$ flexion were 63.3\% (50.8-75.9\%), 68.3\% (56.2-80.5\%), 80.0\% (68.6$90.4 \%)$, and $80.0 \%$ (68.6-90.4\%), respectively.

There was concordance between the SDA and sensor data at all four flexion positions in 22 knees, at 3 positions in 21 knees, at 2 positions in 10 knees, at 1 position in 4 knees, and at 0 positions in 3 knees. A comparison of all 60 knees with the group of 22 knees in which the sensor data showed soft tissue balance at all four positions showed that the compartment pressure was smaller in the sensor-balanced group than in the whole sample. The pressures in the medial and lateral compartments were significantly larger when the knee was in extension than flexion and decreased as the knee became more flexed (Fig. 2).

\section{Agreement between the GB and sensor data}

The GB assessment was consistent with the sensor data in 37 knees at $0^{\circ}$ and in 37 knees at $90^{\circ}$, while the GB and sensor data were inconsistent in 9 knees at $0^{\circ}$ and in 6 knees at $90^{\circ}$ (Table 1). The sensitivity of gap balancing was improved using the GB compared with SDA. Interestingly, the sensor data showed that the ICPD in knees balanced using the GB exceeded the value suggested by the manufacturer in some cases. The gap difference was larger than $1 \mathrm{~mm}$ (range 2-6 mm) at $0^{\circ}$ in five knees, and larger than $4 \mathrm{~mm}$ (range -5 to 0 or $6 \mathrm{~mm}$ ) at $90^{\circ}$ in eight knees.

Table 2 outlines the GB outcomes at each position. There were no differences between the GB measurements in extension and flexion. The accuracy, sensitivity, and positive predictive value of the GB measurements were higher than those of the SDA measurements. However, the specificity and negative predictive value of the GB measurements were not ideal. Cohen's kappa 


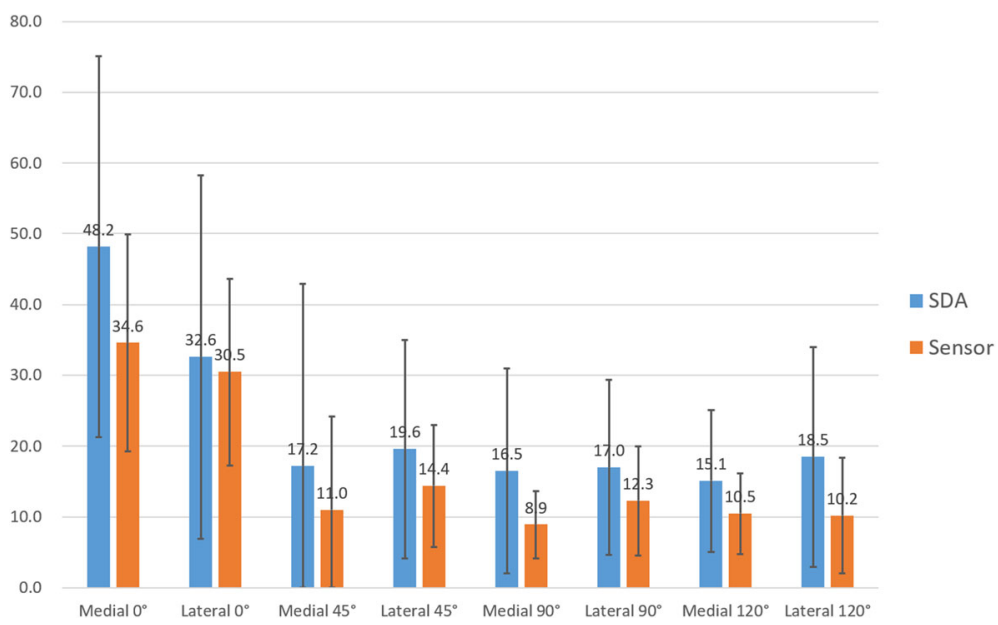

Fig. 2 Compartmental pressure in the SDA group (60 knees) versus the sensor-balanced group (22 knees) at four knee flexion angles. SDA, surgeon-defined assessment

coefficient for the accuracy of the GB measurements compared with the sensor data was 0.406 at $0^{\circ}$ (indicating moderate agreement) and 0.227 at $90^{\circ}$ (indicating fair agreement).

\section{CUSUM analysis}

Figure 3 shows the learning curve for the sensor technology. The CUSUM at $0^{\circ}$ flexion showed a downward trend from the 12th case onwards, although some cases were unbalanced; by case 45 , the curve achieved GPP. The CUSUM at $90^{\circ}$ and $120^{\circ}$ showed successes and an occasional unbalanced downward trend from the 34th and 51st case onwards, respectively; however, these two curves never achieved GPP in all 60 cases. The CUSUM at $45^{\circ}$ showed overall deterioration (upward slope), reaching PPP at case 8 . Although there was a downward trend during this period, the curve continually fluctuated and did not fall below the line indicating PPP; the curve increased dramatically from the 28th case onwards, with attempts at correction after case 45 (downward slope).

\section{Clinic outcomes}

The comparison of KSS scores and WOMAC scores of patients preoperatively and postoperatively is shown in Table 3. The average follow-up period was 12.6 months (6-22 months). The postoperative KSS scores and

Table 1 Consistency of the GB and sensor data

\begin{tabular}{lll}
\hline $\mathbf{0}^{\circ} \mathbf{1 9 0 ^ { \circ }}$ & Sensor balance & Sensor unbalance \\
\hline GB balance & $37 / 37$ & $9 / 9$ \\
GB unbalance & $5 / 8$ & $9 / 6$ \\
\hline
\end{tabular}

GB gap-balancer
WOMAC scores were significantly improved compared with preoperative $(P<0.01)$.

\section{Discussion}

TKA is a very successful orthopedic operation. However, although there is a relatively established system for TKA, about $20 \%$ of patients are not satisfied with the surgical outcome [2]. Soft tissue balancing helps to achieve a successful outcome. Surgeons have traditionally relied on SDA for soft tissue balancing, with few objective assessments. New electronic sensor technology provides real-time objective data intraoperatively to help determine the gap balance, giving a better TKA outcome $[1,10,11]$. However, it is unclear whether the gap balance achieved using SDA is improved by the use of a $\mathrm{GB}$, and whether the sensor improves the SDA of gap balance (i.e., serves as a training tool for surgeons).

Regarding the soft tissue balance in TKA, previous studies mainly focused on the comparison of SDA and sensor data $[6,12]$. MacDessi et al. [6] prospectively analyzed 322 patients undergoing TKA and demonstrated that the accuracy of SDA compared with the sensor was $63 \%, 57.5 \%$, and $63.8 \%$ at $10^{\circ}, 45^{\circ}$, and $90^{\circ}$, respectively, and that SDA had an overall sensitivity of $81 \%$ and specificity of $37.7 \%$; they concluded that SDA is a poor predictor of the true soft tissue balance when compared with sensor data, particularly in assessing whether a knee is unbalanced. However, Elmallah et al. [12] compared 12 patients who underwent sensor-balanced TKA with 12 patients who underwent manual gap-balanced TKA by an experienced surgeon, and noted high rates of unbalanced knees that were not initially identified using the sensor-guided balance assessment. 
Table 2 Accuracy of gap-balancer measurements compared with sensor data at two knee flexion angles

\begin{tabular}{llll}
\hline Statistical measure of performance & $\mathbf{0}^{\circ}$ mean $\left.\mathbf{( 9 5 \%} \mathbf{C l}\right)$ & $\mathbf{9 0}^{\circ}$ mean $\mathbf{( 9 5 \% ~ C l )}$ & $\begin{array}{l}\boldsymbol{P} \text { values } \\
\text { (angle comparison) }\end{array}$ \\
\hline Accuracy & $76.7 \%(65.7 \% \sim 87.7 \%)$ & $71.7 \%(59.9 \sim 83.4 \%)$ & 0.536 \\
Sensitivity & $88.1 \%(75.0 \% \sim 94.8)$ & $82.2 \%(68.7 \sim 90.7 \%)$ & 0.448 \\
Specificity & $50.0 \%(29.0 \% \sim 70.9 \%)$ & $40.0 \%(19.8 \sim 64.3 \%)$ & 0.580 \\
PPV & $80.4 \%(68.5 \% \sim 92.4)$ & $80.4 \%(68.5 \sim 92.4 \%)$ & 1.000 \\
NPV & $64.2 \%(35.6 \sim 93.0)$ & $42.9 \%(13.2 \sim 72.5 \%)$ & 0.272 \\
\hline
\end{tabular}

Cl confidence interval, $P P V$ positive predictive value, $N P V$ negative predictive value

In our study, the sensitivity of SDA was low at $0^{\circ}$ because the surgeon aimed to acquire a stable knee and made the medial compartment slightly tighter than the lateral compartment. In addition, it was difficult to visualize the gap during valgus examination due to the presence of the patella and fat pad.

The present study showed that the pressures of the medial and lateral compartments after SDA were similar to those of the normal knee joint; that is, the ICPD was the largest at $0^{\circ}$, and the medial pressure was greater than the lateral pressure. As the knee was flexed, the pressure rapidly decreased. However, in the process of knee flexion from $45^{\circ}$ to $120^{\circ}$, the pressure of the knee compartments tended to remain stable; that is, the pressure did not further decrease with the increase in the flexion angle.

Verstraete et al. [13] reported mean pressures of 114 $\mathrm{N}$ and $63 \mathrm{~N}$, respectively, in the medial and lateral compartments at $0^{\circ}$ flexion in eight non-arthritic cadavers; both the medial and lateral compartment pressures decreased rapidly from $0^{\circ}$ to $10^{\circ}$ knee flexion and stabilized after $20^{\circ}$, while the lateral pressure decreased slowly. The pressures in the medial and lateral compartments were only $30 \mathrm{~N}$ and $5 \mathrm{~N}$ at $90^{\circ}$ flexion [13]. However, the absolute forces transmitted through the medial compartment were greater than the loads in the lateral compartment [13]. Risitano et al. [14] defined stable knees as those with a pressure of $50 \pm 20 \mathrm{lb}$ in the medial compartment, $35 \pm 20 \mathrm{lb}$ in the lateral compartment, and a mediolateral intercompartmental difference within $15 \pm$ $5 \mathrm{lb}$, with the medial compartment slightly tighter than the lateral compartment.

Our study found that soft tissue balancing using the electronic sensor achieved a lower compartmental pressure compared with balancing performed using SDA alone. This may be related to the use of the GB and sensor to release the soft tissue again after SDA. Furthermore, in accordance with the sensor-assessed balance, we adjusted the pressure if it was greater than $100 \mathrm{~N}$. Elmallah et al. [12] also found that the use of an electronic sensor resulted in additional soft tissue releases (outside of the surgeon's standard releases), which resulted in lower intercompartmental pressures.

As far as we know, no previous study has compared the GB and electronic sensor data. The GB is different to SDA, as the GB achieves a constant opening force

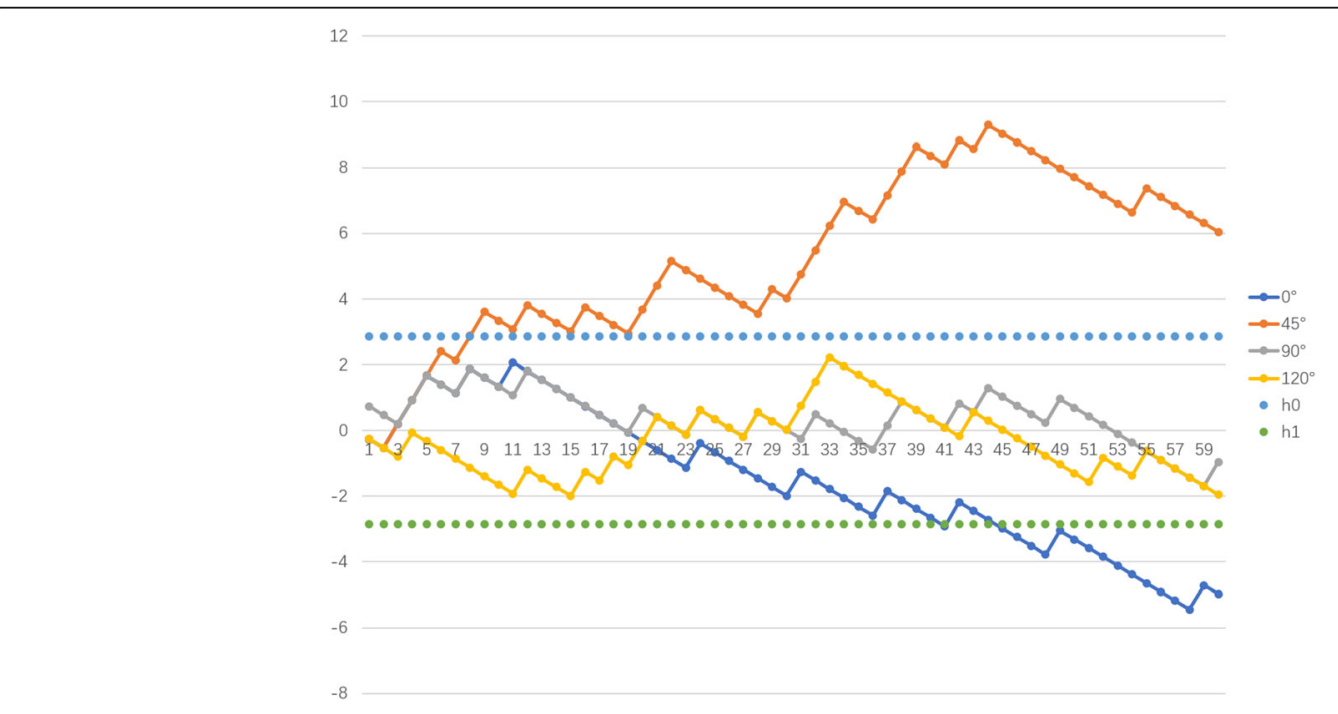

Fig. 3 Combined continuous cumulative summation chart. ho, the unacceptable control line; h1, the acceptable control line 
Table 3 Comparison of KSS scores and WOMAC scores preoperatively and postoperatively

\begin{tabular}{llll}
\hline & Preoperative (mean \pm SD) & Postoperative (mean \pm SD) & $P$ values \\
\hline KSS functional score & $66.6 \pm 14.0$ & $94.3 \pm 6.8$ & $<0.01$ \\
KSS clinical score & $49.3 \pm 12.5$ & $84.4 \pm 13.9$ & $<0.01$ \\
KSS total score & $116.0 \pm 23.6$ & $178.7 \pm 19.2$ & $<0.01$ \\
WOMAC & $47.2 \pm 8.5$ & $10.5 \pm 7.7$ & $<0.01$ \\
\hline
\end{tabular}

accurate to $1 \mathrm{~mm}$. Thus, the GB is more accurate than SDA, especially at $0^{\circ}$. However, the specificity of the GB was relatively low, suggesting that the GB has a poor ability to detect unbalanced knees. Our study showed that the GB had good accuracy at $0^{\circ}$, but that the accuracy decreased with knee flexion, similarly to the accuracy of SDA. MacDessi et al. [6] reported that SDA was a poor predictor of knee imbalance, particularly as the knee flexion angle increases, with a sensitivity twice as large as the specificity.

In our study, some sensor-balanced knees had a larger gap difference between the medial and lateral compartments than that recommended by the manufacturer of the GB. This may be because the sensor only considers the ICPD. Therefore, although the interventricular difference was within the normal range, the medial and lateral pressure distribution did not conform to the physiological state. In a setting that emulates the surgical environment, an intact knee specimen under normal and native ligament tension creates a larger compressive load on the medial side than the lateral side [13], while the sensor does not consider this standard. To better simulate the characteristics of knee joint pressure distribution under physiological conditions, Risitano et al. [14] proposed that the balance standard of the electronic sensor should have a greater pressure in the medial compartment than the lateral compartment; that is, a medial minus lateral pressure of $10-20 \mathrm{lb}$, rather than the commonly used pressure difference of $\pm 15 \mathrm{lb}$. According to our data, the pressure was $10-40 \mathrm{~N}$ greater in the medial compartment than the lateral compartment at $0^{\circ}$.

As a novel technology, the electronic sensor has a learning curve. Surgeons need to become proficient in using the sensor to adapt to the new technology. Training objectives include minimizing the operation time and accurately adjusting the unbalanced pressure. Lakra et al. [15] analyzed 287 consecutive patients who underwent TKA using an electronic sensor to achieve gap balance. It took approximately 41 cases of sensor-assisted TKA to reduce the operation time of the sensor-based surgery from $120 \mathrm{~min}$ to $109 \mathrm{~min}$, which was close to the conventional operation time [15]. This suggests that surgeons master the use of sensor technology after 41 TKA cases. Woon et al. [9] used CUSUM to evaluate the learning curve of TKA with an electronic sensor. The data of $10^{\circ}, 45^{\circ}$, and $90^{\circ}$ were analyzed. During the non-blinded Phase I, the CUSUM $10^{\circ}$ reached GPP at case 41 , the CUSUM $90^{\circ}$ showed a downward trend but never achieved GPP, and the CUSUM $45^{\circ}$ achieved PPP at case 11 [9]. However, MacDessi et al. [16] used a chisquared learning curve histogram and found that the capacity of SDA to determine balance compared with sensor data did not improve with ongoing use of the sensor.

Our results showed that the learning curve for the sensor technology was the shortest at $0^{\circ}$, followed by $90^{\circ}$ and $120^{\circ}$, while $45^{\circ}$ was the most difficult, which was similar to the findings of Woon et al. [9]. This may be because the extension balance of TKA is more important than the flexion balance. In TKA, the surgeon should first assess the extension gap balance to ensure excellent lower limb alignment, then assess the gap balance at $90^{\circ}$ flexion, and finally assess the balance at $45^{\circ}$ and $120^{\circ}$. In addition, the soft tissue balance at different knee flexion angles affects the balance at other angles; the soft tissue release at one angle sometimes leads to a change in the ICPD at another angle. In such cases, surgeons may need to compromise and prioritize the balancing of the extension gap.

The present study has some limitations. First, the sample size was relatively small, especially in the CUSUM analysis. While post hoc sample size estimation showed: according to the $76.6 \%$ agreement between GB and sensor group, the allowable error is $10 \%$. The $95 \%$ CI of the agreement rate was estimated by PASS 14.0, and 77 knees needed to be studied. The sample size of this study is 60 knees, while the gap is acceptable. It may have been better to cross the learning curve at $90^{\circ}$ and $120^{\circ}$ in the later cases. Second, the acceptable limits of the ICPD are not clear. Previous research has showed that a maximum ICPD of 15 psi may be an inappropriate definition of knee balance, and that the ICPD should be 0-40 psi [6, 17]. Furthermore, Meneghini et al. [18] showed that the intercompartmental force difference did not support the categorical mediolateral force difference of $\leq 15 \mathrm{lb}$. In knees balanced using the sensor, we found a similar compartmental pressure to that reported by Verstraete et al. [13]. Our study defined knee balance as an ICPD of $30 \mathrm{~N}$ at each flexion angle, which is the standard provided by the sensor manufacturer. However, the optimal target ligament balance for each patient undergoing TKA remains unknown. Future studies are 
required to evaluate the differences in functional outcomes between these patient cohorts and to assess the role of different load measurements [16]. Third, we conducted early period follow-up on the postoperative function of the patients. It is obvious that the knee functions after TKA are significantly improved. However, our study did not compare with the postoperative follow-up of non-sensor groups, which is also a limitation of our study. Fourth, we only included one surgeon, and its advantage is to avoid the errors caused by surgical techniques and measurement methods. However, we cannot effectively count the differences between surgeons and measured the interclass correlation coefficient

\section{Conclusion}

In the present study, SDA was a poor predictor of knee balance. The use of a GB improved the accuracy of gap balancing, but there was still room for improvement, particularly when assessing knees that were unbalanced. SDA showed a learning effect with the ongoing use of the sensor at all knee positions except $45^{\circ}$, which was the most difficult position to balance. Although the sensor is the gold standard, the optimal ICPD for each patient undergoing TKA remains unknown. Further study is necessary to compare the outcome among the patient cohorts with different ICPDs.

\section{Abbreviations}

TKA: Total knee arthroplasty; SDA: Surgeon-defined assessment; GB: Gapbalancer; CUSUM: Cumulative summation; GPP: Good prior performance; PPP: Poor prior performance

\section{Acknowledgements}

We thank Kelly Zammit, BVSc, from Liwen Bianji, Edanz Editing China (www liwenbianji.cn/ac), for editing the English text of a draft of this manuscript.

\section{Authors' contributions}

Ran Zhao: data collection and management, data analysis, manuscript writing. Liyuan TAO: data analysis. Yanqing Liu: project development, manuscript editing. Hua Tian: data management, project development. All authors read and approved the final manuscript.

\section{Funding}

This work was supported by a grant from the Beijing Municipal Science \& Technology Commission (No. D171100003217001).

\section{Availability of data and materials}

The datasets obtained and/or analyzed during the current study are available from the corresponding author on reasonable request.

\section{Declarations}

\section{Ethics approval and consent to participate}

The present study titled "Accuracy of soft tissue balancing in total knee arthroplasty using surgeon-defined assessment versus a gap-balancer or electronic sensor" was approved by our ethics committee. Please refer to the supplementary material. All patients provided written informed consent before undergoing total knee arthroplasty.

\section{Consent for publication}

All patients provided written informed consent for the use of their data for research purposes.

\section{Competing interests}

The authors declare that they have no competing interests.

Received: 22 January 2021 Accepted: 20 April 2021

Published online: 08 May 2021

References

1. Golladay GJ, Bradbury TL, Gordon AC, Fernandez-madrid IJ, Krebs VE, Patel PD, et al. Are patients more satisfied with a balanced total knee arthroplasty? J Arthroplast. 2019;34(7):S195-s200. https://doi.org/10.1016/j.a rth.2019.03.036.

2. Gustke KA, Golladay GJ, Roche MW, Jerry GJ, Elson LC, Anderson CR. Increased satisfaction after total knee replacement using sensor-guided technology. Bone Joint J. 2014;96-b:1333-8.

3. Wylde $V$, Hewlett $S$, Learmonth ID, Dieppe $P$. Persistent pain after joint replacement: prevalence, sensory qualities, and postoperative determinants. Pain. 2011;152(3):566-72. https://doi.org/10.1016/j.pain.2010.11.023.

4. Lombardi AV Jr, Berend KR, Adams JB. Why knee replacements fail in 2013: patient, surgeon, or implant? Bone Joint J. 2014;96-b:101-4.

5. Griffin FM, Insall JN, Scuderi GR. Accuracy of soft tissue balancing in total knee arthroplasty. J Arthroplast. 2000;15(8):970-3. https://doi.org/10.1054/a rth.2000.6503.

6. Macdessi SJ, Gharaibeh MA, Harris IA. How Accurately Can Soft Tissue Balance Be Determined in Total Knee Arthroplasty? J Arthroplast. 2019;34: 290-4 e291.

7. Viera AJ, Garrett JM. Understanding interobserver agreement: the kappa statistic. Fam Med. 2005;37:360-3.

8. Khan N, Abboudi H, Khan MS, Dasgupta P, Ahmed K. Measuring the surgical 'learning curve': methods, variables and competency. BJU Int. 2014;113(3): 504-8. https://doi.org/10.1111/bju.12197.

9. Woon CYL, Carroll KM, Lyman S, Mayman DJ. Dynamic sensor-balanced knee arthroplasty: can the sensor "train" the surgeon? Arthroplast Tod. 2019; 5(2):202-10. https://doi.org/10.1016/j.artd.2019.03.001.

10. Chow JC, Breslauer L. The Use of Intraoperative Sensors Significantly Increases the Patient-Reported Rate of Improvement in Primary Total Knee Arthroplasty. Orthopedics. 2017:40(4):e648-51. https://doi.org/10.3928/014 77447-20170503-01.

11. Gustke KA, Golladay GJ, Roche MW, Elson LC, Anderson CR. Primary TKA patients with quantifiably balanced soft-tissue achieve significant clinical gains sooner than unbalanced patients. Advanc Orthop. 2014:628695.

12. Elmallah RK, Mistry JB, Cherian JJ, Chughtai M, Bhave A, Roche MW, et al. Can We Really "Feel" a Balanced Total Knee Arthroplasty? J Arthroplast. 2016;31(9):102-5. https://doi.org/10.1016/j.arth.2016.03.054.

13. Verstraete MA, Meere PA, Salvadore G, Victor J, Walker PS. Contact forces in the tibiofemoral joint from soft tissue tensions: Implications to soft tissue balancing in total knee arthroplasty. J Biomech. 2017:58:195-202. https://doi. org/10.1016/j.jbiomech.2017.05.008

14. Risitano S, Karamian B, Indelli PF. Intraoperative load-sensing drives the level of constraint in primary total knee arthroplasty: Surgical technique and review of the literature. J Clin Orthop Trauma. 2017:8(3):265-9. https://doi. org/10.1016/j.jcot.2017.06.004.

15. Lakra A, Sarpong NO, Jennings EL, Grosso MJ, Cooper HJ, Shah RP, et al. The Learning Curve by Operative Time for Soft Tissue Balancing in Total Knee Arthroplasty Using Electronic Sensor Technology. J Arthroplast. 2019;34(3): 483-7. https://doi.org/10.1016/j.arth.2018.11.014.

16. MacDessi S. J., Wood J. A., Diwan A. D., Harris I. A. Surgeon-defined assessment is a poor predictor of knee balance in total knee arthroplasty: a prospective, multicenter study, Knee surgery, sports traumatology, arthroscopy : official journal of the ESSKA 2020.

17. Gustke KA, Golladay GJ, Roche MW, Elson LC, Anderson CR. A new method for defining balance: promising short-term clinical outcomes of sensorguided TKA. J Arthroplast. 2014;29(5):955-60. https://doi.org/10.1016/j.arth.2 013.10.020.

18. Meneghini RM, Ziemba-Davis MM, Lovro LR, Ireland PH, Damer BM. Can intraoperative sensors determine the "target" ligament balance? early outcomes in total knee arthroplasty. J Arthroplast. 2016;31(10):2181-7. https://doi.org/10.1016/j.arth.2016.03.046.

\section{Publisher's Note}

Springer Nature remains neutral with regard to jurisdictional claims in published maps and institutional affiliations. 